\title{
The expanding network of redox signaling: new observations, complexities, and perspectives
}

Roy J. Soberman

Original citation: J. Clin. Invest. 111:571-574 (2003). doi:10.1172/JCI200318099.

Citation for this corrigendum: J. Clin. Invest. 111:1093 (2003). doi:10.1172/JCI200318099C.

During the final stages of production, an error was introduced into the list of nonstandard abbreviations and into the text. The correct list of nonstandard abbreviations and the correct sentence appear below.

Nonstandard abbreviations used: reactive oxygen species (ROS); hydrogen peroxide $\left(\mathrm{H}_{2} \mathrm{O}_{2}\right)$; hypoxia-inducible factor 1 (HIF-1); carboxy-terminal binding protein (CtBP); apoptosis-inducing factor (AIF); apoptosis signal-regulating kinase 1 (ASK1).

Sensing nuclear oxygen tension. Since its original description, the study of the transcription factor hypoxia-inducible factor 1 (HIF-1) has demonstrated its central role in regulating the body's response to changing oxygen levels (4-6). 